\author{
Andrzej STUDZIŃSKI ${ }^{1}$ \\ Katarzyna PIETRUCHA-URBANIK ${ }^{2}$
}

\title{
PREVENTIVE MAINTENANCE AND RELIABILITY OF WATER SUPPLY SYSTEM ELEMENTS
}

\begin{abstract}
One of the main requirements for municipal systems, particularly water supply systems is their reliable operation. Water supply systems are operated continuously for a long time. Therefore, it building elements are also subject to intense and continuous operation. Human intervention detects and removes the problems arising as a potential source of failure. A common solution to the problem are preventative renovations. The term recovery is meant a preventive action to reduce the rate of loss of efficiency. Preventive renovations do not eliminate the possibility of damage, but can reduce the likelihood of emergency renew. Renew prevention strategy it is the appropriate location of their time in order to achieve maximum profitability of the project or the required level of reliability. The most common strategies are used periodically after a predetermined time. In this work mathematical model of preventive renovations was presented and it's following conclusions. It was shown that in case of exponential distribution of failures scheduled maintenances do not increase reliability of an element. Scheduled maintenances extend an expected value of working time if next working times are shorten, for instance if it's possible to describe them by Weibull distribution with it's parameter $\alpha>1$.
\end{abstract}

Keywords: reliability, working time, maintenance, water supply system

\section{Introduction}

From reliability point of view there are two kinds of elements: repairable after a failure are repaired and become fit, and unrepairable, which have to be exchanged into a new ones [14]. Elements, which built water supply systems are mostly repairable, like pipes, pumps or water reservoirs. Failure cause economic consequences for the water supply company resulting in the reduction or interruption of water supply $[10,12]$. Prevention strategy constitute of the appropriate location as to achieve maximum project profitability or the required reliability level $[8,9,11]$.Their life cycle shown on fig. 1 , consists of a three basis phas-

\footnotetext{
${ }^{1}$ Autor do korespondencji/corresponding author: Andrzej Studziński, Politechnika Rzeszowska, al. Powstańców Warszawy 6, tel. 17 8651703, astud@prz.edu.pl

2 Katarzyna Pietrucha-Urbanik, Politechnika Rzeszowska, al. Powstańców Warszawy 6, tel. 17 8651703,kpiet@prz.edu.pl
} 
es: initial phase during start-up, useful life phase and wearout phase, which ends technical life of an element $[1,4,6]$.

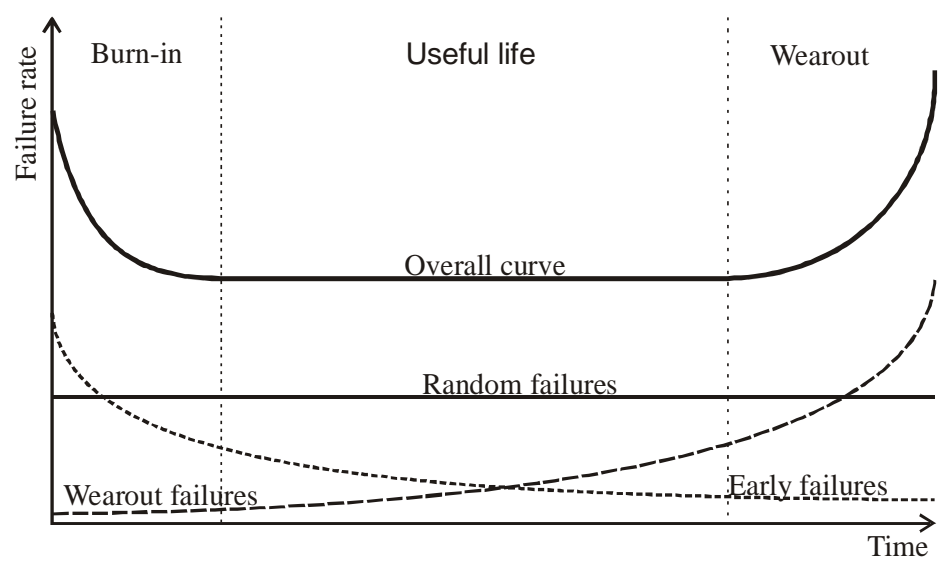

Fig. 1. Life cycle of an element ("bathtube shape curve") on the basis of [6]

Rys. 1. Uszkodzenie element (krzywa wannowa) na podstawie pracy [6]

The most important is useful life time, usually lasting from a dozen or so till a few dozen years, for instance water pipe useful life time is estimated at 50 years $[3,7]$. The useful life consist of one after another working times $T_{p}$ and restoration times $T_{n}$ - since moment of failure till beginning an operation, as shown on fig. 2.

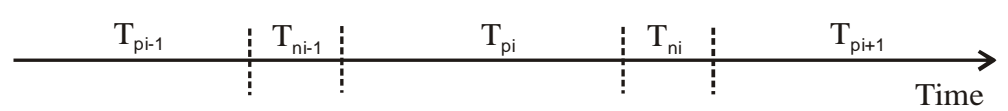

Fig. 2. Useful life time of a repairable element, Tp - working time, Tn - restoration time

Rys. 2. Proces eksploatacji elementu odnawialnego: Tp - czas pracy, Tn - czas odnowy

\section{Mathematical model of preventive renovations}

Life cycle of an element consists of one after another working times $\mathrm{T}_{\mathrm{pi}}$ and restoration times $T_{n i}$. Let $T_{p}$ be a random variable of working time of an element, $T_{z}$ is a random variable of time till the next preventive renovation. Working time till the next renovation $\mathrm{T}$ is a random variable $[1,5]$ :

$$
\mathrm{T}=\min \left\{\mathrm{T}_{\mathrm{p}}, \mathrm{T}_{\mathrm{z}}\right\}
$$

If random variables $T_{p} i T_{z}$ are independent cumulative distribution function of up time $\mathrm{F}_{\mathrm{T}}$ is: 


$$
\mathrm{F}_{\mathrm{T}}(\mathrm{t})=\mathrm{F}_{\mathrm{Tp}}(\mathrm{t})+\mathrm{F}_{\mathrm{Tz}}(\mathrm{t})-\mathrm{F}_{\mathrm{Tp}}(\mathrm{t}) \cdot \mathrm{F}_{\mathrm{Tz}}(\mathrm{t})
$$

When time between preventive renovations $t_{z}$ is determined and the renovations are made in fixed intervals, as shown on fig. 3, cumulative distribution function is:

$$
F_{T}(t)=\left\{\begin{array}{c}
0 \text { dla } t \leq 0 \\
R_{T p}(t) \text { dla } 0<t \leq t z \\
1 \text { dla } t>t z
\end{array}\right.
$$

where: $R_{T p}-$ is reliability of the element.

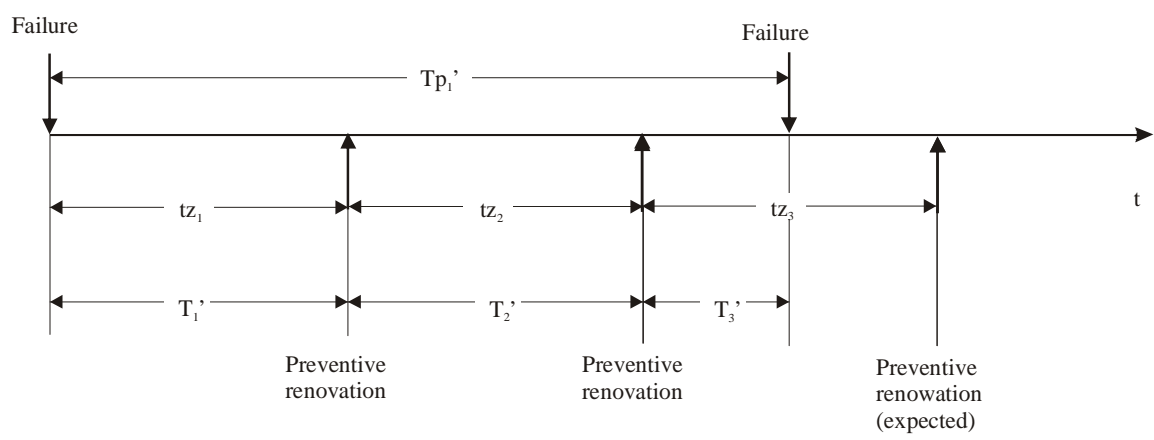

Fig. 3. Useful life time of a repairable element with preventive renovations, $T_{p}-$ working time, $T_{z}-$ time between preventive renovations, $\mathrm{T}_{1}{ }^{\prime}=\min \left\{\mathrm{T}_{\mathrm{p} 1}{ }^{\prime} ; \mathrm{t}_{\mathrm{z} 1}\right\}=\mathrm{t}_{\mathrm{z} 1} \Rightarrow \mathrm{X}_{1}=0 ; \mathrm{Y}_{1}=\mathrm{X}_{1}=0$ what means that till the first renovation there was no failure; $\mathrm{T}_{2}{ }^{\prime}=\min \left\{\mathrm{T}_{\mathrm{p} 1}{ }^{\prime} ; \mathrm{t}_{\mathrm{z} 2}\right\}=\mathrm{t}_{\mathrm{z} 2} \Rightarrow \mathrm{X}_{2}=0 ; \mathrm{Y}_{2}=$ $X_{1}+X_{2}=0$ what means that till the second renovation there was no failure; $T_{3}{ }^{\prime}=\min \left\{T_{p 1}{ }^{\prime} ; t_{z 3}\right\}=$ $\mathrm{T}_{\mathrm{p} 1}{ }^{\prime} \Rightarrow \mathrm{X}_{3}=1 ; \mathrm{Y}_{3}=\mathrm{X}_{1}+\mathrm{X}_{2}+\mathrm{X}_{3}=1$ what means that till the third renovation there was 1 failure; etc.

Rys. 3. Proces eksploatacji elementu odnawialnego z profilaktycznymi odnowami, $\mathrm{T}_{\mathrm{p}}$ - czas pracy, $\mathrm{T}_{\mathrm{z}}$ - czas pomiędzy profilaktycznymi odnowami, $\mathrm{T}_{1}{ }^{\prime}=\min \left\{\mathrm{T}_{\mathrm{p} 1}{ }^{\prime} ; \mathrm{t}_{\mathrm{z} 1}\right\}=\mathrm{t}_{\mathrm{z} 1} \Rightarrow \mathrm{X}_{1}=0 ; \mathrm{Y}_{1}=\mathrm{X}_{1}$ $=0$ co oznacza, że do czasu pierwszej odnowy nie było awarii; $\mathrm{T}_{2}{ }^{\prime}=\min \left\{\mathrm{T}_{\mathrm{p} 1}{ }^{\prime} ; \mathrm{t}_{\mathrm{z} 2}\right\}=\mathrm{t}_{\mathrm{z} 2} \Rightarrow \mathrm{X}_{2}=$ $0 ; \mathrm{Y}_{2}=\mathrm{X}_{1}+\mathrm{X}_{2}=$ co oznacza, że do czasu drugiej odnowy nie było awarii; $\mathrm{T}_{3}{ }^{\prime}=\min \left\{\mathrm{T}_{\mathrm{p} 1}{ }^{\prime} ; \mathrm{t}_{\mathrm{z} 3}\right\}=$ $\mathrm{T}_{\mathrm{p} 1}{ }^{\prime} \Rightarrow \mathrm{X}_{3}=1 ; \mathrm{Y}_{3}=\mathrm{X}_{1}+\mathrm{X}_{2}+\mathrm{X}_{3}=1$ co oznacza, że do czasu trzeciej odnowy wystąpiła jedna awaria; etc.

Estimated value of working time till the next renovation $\mathrm{T}-\mathrm{E}(\mathrm{T})$ is:

$$
\mathrm{E}(\mathrm{T})=\int_{0}^{\mathrm{tz}} \mathrm{R}_{\mathrm{Tp}}(\mathrm{t}) \mathrm{dt}
$$


Let random variables $\mathrm{T}_{\mathrm{p} 1}, \mathrm{~T}_{\mathrm{p} 2}, \ldots$ form renovation stream, and a sequence $t_{z 1}, t_{z 2}, \ldots$ be moments of preventive renovations, $t_{z i}$ is a time since $i-1$ till i preventive renovation. Renovation stream is:

$$
\mathrm{T}_{1}, \mathrm{~T}_{1}+\mathrm{T}_{2}, \mathrm{~T}_{1}+\mathrm{T}_{2}+\mathrm{T}_{3}, \ldots
$$

where:

$$
\mathrm{T}_{\mathrm{i}}=\min \left\{\mathrm{T}_{\mathrm{p}_{\mathrm{i}}}, \mathrm{t}_{\mathrm{z}_{\mathrm{i}}}\right\}
$$

Assign renovation stream (5) to a sequence of Bernoullie's binary random variables $\mathrm{X}_{1}, \mathrm{X}_{2}, \ldots$ that:

$$
X_{i}=\left\{\begin{array}{l}
0 \text { dla } T_{i} \geq t_{i} \\
1 \text { dla } T_{i}<z_{i}
\end{array}\right.
$$

Where value 0 means, that restoration number i was preventive, and value 1 means that the restoration was made after a failure of an element. Random variable $Y_{n}$ is a number of failure renovations till renovation $n$, it was shown at fig. 3:

$$
Y_{n}=\sum_{i=1}^{n} X_{i} \quad \text { for } n=1,2, \ldots
$$

If $\mathrm{T}_{\mathrm{p} 1}$ is a random variable with the same distribution as $\mathrm{T}_{\mathrm{p} 2}, \mathrm{~T}_{\mathrm{p} 3} \ldots$, the probability, that among the next $\mathrm{n}$ restorations is $\mathrm{k}$ restorations after failures can be calculated by following formula:

$$
\mathrm{P}\left(\mathrm{Y}_{\mathrm{n}}=\mathrm{k}\right)=\left(\begin{array}{l}
\mathrm{n} \\
\mathrm{k}
\end{array}\right)[\mathrm{F}(\mathrm{tz})]^{\mathrm{k}} \cdot[1-\mathrm{F}(\mathrm{tz})]^{\mathrm{n}-\mathrm{k}} \quad \text { dla } \mathrm{k}=1,2, \ldots, \mathrm{n}
$$

And the probability that there were no failures $(\mathrm{k}=0)$ is:

$$
\mathrm{P}\left(\mathrm{Y}_{\mathrm{n}}=0\right)=[1-\mathrm{F}(\mathrm{tz})]^{\mathrm{n}}
$$

Tp' time of renovated preventive element includes a number of intervals between preventive renovations and a time between the last preventive renovation and a failure. The probability that the element will not suffer damage till time $t>0$ with periodic strategy of preventive renovations is:

$$
\mathrm{P}(\mathrm{t})=\mathrm{R}_{\mathrm{Tp}}{ }^{\mathrm{n}}(\mathrm{tz}) \cdot \mathrm{R}_{\mathrm{Tp}}(\mathrm{t}-\mathrm{n} \cdot \mathrm{tz})
$$


where: $\mathrm{n}=\frac{\mathrm{t}}{\mathrm{t}_{\mathrm{z}}}$ is a number of working time intervals - natural number no less than $\frac{\mathrm{t}}{\mathrm{t}_{\mathrm{z}}}$. is:

Expected value of time till failure with preventive renovations $\mathrm{T}_{\mathrm{op}}, \mathrm{E}\left(\mathrm{T}_{\mathrm{op}}\right)$

$$
E(T o p)=\frac{1}{1-R_{T p}(t z)} \cdot \int_{0}^{t z} R_{T p}(t) d t
$$

\section{Mathematical model of ageing element}

For ageing elements random variable of working time $T_{p}$ Weibull distribution is applied. For elements that built water supply systems usually exponential distribution is used, which is a special case of Weibull distribution. Reliability of an element can be calculated according to equations (13-17). Probability density function is [1]:

$$
\mathrm{f}(\mathrm{t})=\lambda \cdot \alpha \cdot \mathrm{t}^{\alpha-1} \cdot \exp \left(-\lambda \cdot \mathrm{t}^{\alpha}\right) \quad \text { dla } \mathrm{t}>0
$$

Failure rate $\lambda(t)$ is:

$$
\lambda(\mathrm{t})=\lambda \cdot \alpha \cdot \mathrm{t}^{\alpha-1}
$$

Cumulative distribution function of up time is:

$$
\mathrm{F}(\mathrm{t})=1-\exp \left(-\lambda \cdot \mathrm{t}^{\alpha}\right)
$$

Reliability function $\mathrm{R}(\mathrm{t})$ is:

$$
R(t)=\exp \left(-\lambda \cdot t^{\alpha}\right)
$$

Expected value of working time $T_{p}-E\left(T_{p}\right)$ is:

$$
\mathrm{E}(\mathrm{Tp})=\Gamma\left(1+\frac{1}{\alpha}\right) \cdot \lambda^{-\frac{1}{\alpha}}
$$

where: $\alpha$-Weibull distribution parameter.

Parameter $\alpha$ describes probability of failure during ageing of an element:

- $\alpha<1$ function $\lambda(\mathrm{t})$ is monotone decreasing - it means that probability of failure is decreasing in time passage, it can suggest that elements in population have manufacturing defects, 
- $\alpha=1$ function $\lambda(t)$ is constant - Weibull distribution becomes exponential - means that probability is constant - it implies that cause of failures are random events,

- $\alpha>1$ function $\lambda(\mathrm{t})$ is monotone increasing - the probability of failure is increasing in time, probably because of ageing of elements.

\section{Conclusions}

In this work mathematical model of preventive renovations was presented and it's following conclusions.

It was shown that in case of exponential distribution of failures scheduled maintenances do not increase reliability of an element.

Scheduled maintenances extend an expected value of working time if next working times are shorten, for instance if it's possible to describe them by Weibull distribution with its parameter $\alpha>1$.

The paper shows a model of prevention renovation based on reliability theory.

The model was applied for elements of water supply system. The conclusions are:

- if a damage intensity index of an element was described by an exponential distribution, using prevention renovation would not extend an expected value of element's working time,

- if the index was described by Weibull's distribution with distribution's parameter $\alpha>1$, prophylaxis would extend an expected value of working time.

\section{Literature}

[1] Bobrowski D.: Modele i metody matematyczne w teorii niezawodności, Wydawnictwo Naukowo - Techniczne, Warszawa 1985.

[2] Denczew S.: Modern management of the utilization of water supply and sewage disposal systems. Environmental protection Engineering, vol. 29, 2003, p. 73-80.

[3] Denczew S., Królikowski A.: Podstawy nowoczesnej eksploatacji systemów wodociągowych i kanalizacyjnych. Arkady, Warszawa 2002.

[4] Loganathan G. V., Sherali H. D, Shah M. P.: A two-phase network design heuristic for minimum cost water distribution systems under a reliability constrait. Enineering Optimization., vol. 4, 1990, p. 311-336.

[5] Malik M.: Reliable preventive maintenance scheduling. AIEE Trans, vol. 11, 1979, p. 221-228.

[6] Mays W. L.: Reliability analysis of water distribution systems. American Society of Civil Engineers, New York 1989.

[7] Pascual R., Ortega J. H.: Optimal replacement and overhaul decisions with imperfect maintenance and warranty contracts. Reliability Engineering and System Safety, vol. 91, 2006, p. 241-248.

[8] Pietrucha-Urbanik K.: Prioritizing water pipe renewal using fuzzy set theoryJournal of KONBiN 1(33)2015, p. 243-250. DOI 10.1515/jok-2015-032. 
[9] Rak J.: Metoda planowania remontów sieci wodociągowej na przykładzie miasta Krosna, Czasopismo Inżynierii Lądowej, Środowiska i Architektury, JCEEA, t. XXXI, z. 61 (1/14), 2014, s. 225-232. DOI:10.7862/rb.2014.15.

[10] Rak J.: Podstawy bezpieczeństwa systemów zaopatrzenia w wodę. Monografie Komitetu Inżynierii Środowiska Polskiej Akademii Nauk, vol. 28. Wydawn. Drukarnia Liber Duo Kolor, Lublin 2005.

[11] Rak J.: Systemowe zarządzanie bezpieczeństwem SZZW. Instal, nr 2 (292), 2009, s. 43-48.

[12] Studziński A., Pietrucha-Urbanik K., Mędrala A.: Analiza strat wody oraz awaryjności w wybranych systemach zaopatrzenia w wodę, Czasopismo Inżynierii Lądowej, Środowiska i Architektury, JCEEA, t. XXXI, z. 61 (4/14), 2014, s. 193-201. DOI:10.7862/rb.2014.144.

[13] Tchórzewska-Cieślak B., Rak. R. J.: Propozycja nowej systematyki własności funkcjonowania systemu na przykładzie systemu zaopatrzenia w wodę. Gaz, Woda i Technika Sanitarna, vol. 5, 2008, s. 20-22.

[14] Wieczysty A.: Metody oceny i podnoszenia niezawodności działania komunalnych systemów zaopatrzenia w wodę. Wydawnictwo Komitetu Inżynierii Środowiska PAN, Kraków 2001.

[15] Wieczysty A.: Niezawodność systemów wodociągowych i kanalizacyjnych. Cz. I, II. Politechnika Krakowska, Kraków 1990.

\section{ODNOWY PROFILAKTYCZNE A NIEZAWODNOŚĆ ELEMENTÓW SYSTEMU ZAOPATRZENIA W WODE}

\section{Streszczenie}

Jednym z głównych wymagań stawianych systemom komunalnym, a w szczególności systemowi zaopatrzenia w wodę jest ich niezawodna praca. Systemy zaopatrzenia w wodę są eksploatowane w sposób ciągły w długim przedziale czasowym. W związku z tym budujące go elementy także podlegają intensywnej i długotrwałej eksploatacji. Kontrolna ingerencja człowieka wykrywa i usuwa ich usterki będące potencjalnym źródłem awarii. Powszechnie stosowanym rozwiązaniem stają się tzw. odnowy profilaktyczne. Pod pojęciem odnowy profilaktycznej rozumie się działanie na zdatnym obiekcie, mające na celu zmniejszenie szybkości utraty jego zdolności użytkowej. Odnowy profilaktyczne nie likwidują możliwości powstawania uszkodzeń, ale mogą zmniejszać prawdopodobieństwo wystąpienia odnów awaryjnych. Strategia odnów profilaktycznych polega na odpowiednim usytuowaniu ich w czasie, tak aby osiągnąc maksymalną rentowność przedsięwzięcia lub wymagany poziom niezawodności. Najczęściej stosuje się tzw. strategie periodyczne, które polegają na odnowach profilaktycznych po z góry określonym czasie eksploatacji elementu i odnowie awaryjnej z chwilą jego uszkodzenia. W pracy przedstawiono model matematyczny odnowy profilaktycznej na przykładzie elementów systemu zaopatrzenia w wodę. Wykazano, że w przypadku wystąpienia rozkładu wykładniczego awarii zastosowanie odnów profilaktycznych nie zwiększa niezawodności elementu. Remonty planowe wydłużają wartość oczekiwaną czasu pomiędzy uszkodzeniami jeśli kolejne jego wartości się skracają, co oznacza, że występuje starzenie elementu, które można opisać rozkładem Weibulla z parametrem $\alpha>1$.

Słowa kluczowe: niezawodność, czas pracy bezuszkodzeniowej, obsługa, system zaopatrzenia w wodę 
Przestano do redakcji: 29.05.2015 r.

Przyjęto do druku: 30.10.2015 r.

DOI: $10.7862 / \mathrm{rb} .2015 .126$ 